\title{
Diseño y fabricación de un ROV acuático recogedor de basura marina
}

\section{Design and manufacture of a marine garbage collector aquatic ROV}

Rigoberto Cerna Castro, est. ${ }^{1}$, Mario Aguilar, est. ${ }^{1}$

${ }^{1}$ Universidad Tecnológica Centroamericana, San Pedro Sula, Honduras, rigocerna98@unitec.edu, mariodcr711@unitec.edu 


\title{
Diseño y fabricación de un ROV acuático recogedor de basura marina
}

\section{Design and manufacture of a marine garbage collector aquatic ROV}

\author{
Rigoberto Cerna Castro, est. ${ }^{1}$, Mario Aguilar, est. ${ }^{1}$ \\ ${ }^{1}$ Universidad Tecnológica Centroamericana, San Pedro Sula, Honduras, rigocerna98@unitec.edu, mariodcr711@unitec.edu
}

\begin{abstract}
Resumen-El presente trabajo presenta el diseño y desarrollo de un vehículo tele operado por radio frecuencia de $2.4 G \mathrm{Gz}$, con la habilidad de apoyar al operador con visión artificial consiguiendo detectar objetos implementando una red neuronal en tiempo real. Se entreno una red neuronal por medio del método de cascada para la correcta detección de los objetos que se necesitan observar en pantalla. Este proyecto fue creado para poder combatir la basura proveniente del Rio Motagua en Guatemala hacia las playas de Omoa en Honduras, cabe mencionar que puede ser implementado en cualquier ambiente acuático que se preste con las condiciones adecuadas para su correcto funcionamiento, el prototipo está diseñado para poder recolectar basura flotando en la superficie como lo hacen los plásticos. La implementación del vehículo tele operado reduce la cantidad de esfuerzo e incrementa la capacidad de recolección de desechos con menos personal de limpieza, así mismo, ayuda al sector turismo y al sector salud manteniendo las aguas limpias con menos contaminación y por lo tanto con menos enfermedades. Para esta investigación se tomaron en cuenta las múltiples variabilidadesde movilización, interacción y riesgos que pueden ocurrir al momento de interactuar con el campo de trabajo, así como las áreasen que puede llegar a trabajar el vehiculó de manera eficaz.
\end{abstract}

Palabras clave: Robótica, visión artificial, radio frecuencia, contaminación, redes neuronales.

Abstract- This work presents the design and development of a $2.4 \mathrm{GHz}$ radio frequency tele-operated vehicle, with the ability to support the operator with artificial vision, detecting objects by implementing a neural network in real time. A neural network was trained through the cascade method for the correct detection of the objects that need to be observed on the screen. This project was created to be able to combat the garbage from the Motagua River in Guatemala to the beaches of Omoa in Honduras, it is worth mentioning that it can be implemented in any aquatic environment that is provided with the appropriate conditions for its correct operation, the prototype is designed to be able to collect trash floating on the surface like plastics do. The implementation of the teleoperated vehicle reduces the amount of effort and increases the waste collection capacity with fewer cleaning personnel, likewise, it helps the tourism sector and the health sector by keeping the waters clean with less pollution and therefore with fewer diseases. For this research, the multiple variabilities of mobilization, interaction and risks that can occur when interacting with the work field were taken into account, as well as the areas in which the vehicle can work effectively.

Keywords- Robotics, computer vision, radio frequency, pollution, neural networks.

\section{INTRODUCCION}

La contaminación de los ambientes acuáticos incrementa cada año de manera muy rápida debido a la producción de plástico a lo largo del planeta, se estima que desde el año 1950 la producción mundial del plástico ha incrementado un $9 \%$ y muchos de los desechos plásticos terminan en nuestros ecosistemas ya sean terrestres o marítimos.[1] El presente trabajo está enfocado en la cuenca del rio motagua, mismo que funciona como principal medio de transporte para los desechos de varias ciudades del país que terminan desembocando en el mar y gran parte en las zonas más turísticas de Omoa, las cuales son unas de las playas más visitadas en Honduras, cabe mencionar que el rio es el más largo y de mayor cuenca en Guatemala con una longitud de $486 \mathrm{~km}$ y una cuenca total de $12.670 \mathrm{~km}^{2}[2]$.

Continuamente, el mundo se enfrenta ante la amenaza del deterioro de varios ecosistemas, en especial el marino, debido la falta de propuestas y resoluciones para detener o incluso disminuir el impacto de los residuos en todo este hábitat. Según las últimas estimaciones basadas en modelos, la mayor parte de los plásticos fluviales se emiten en el continente asiático [3]. Sin embargo, debido a la falta de observaciones, la cantidad exacta de emisiones de plástico de los ríos aún es incierta. El número de estudios de campo que se realizan es pequeño, e incluso, aun así, varios resultados se centran en los ríos de Europa y América del Norte y utilizan una variedad de métodos de recopilación de datos. Debido a esta amenaza, es preciso encontrar una manera eficiente y, sobre todo, accesible para países de Latinoamérica como Honduras para que puedan atacar este problema.

Este problema, que día a día se acelera, es un completo $19^{\text {th }}$ LACCEI International Multi-Conference for Engineering, Education, and Technology: "Prospective and trends in technology and skills for sustainable social development" "Leveraging emerging technologies to construct the future", Buenos Aires -Argentina, July 21-23, 2021. 
desafío ya que constantemente la economía aumenta, la población es creciente y la urbanización de los lugares

debe ser aplicado de manera indefectible. Sin embargo, esto es un problema a nivel mundial porque, incluso con estudios, todas estas medidas cambian o varían mucho de un lugar a otro. Lo que puede ser bien regulado en una parte del mundo, puede ser algo totalmente distinto en otra parte. La gestión de los residuos, la regulación del uso del plástico y el consumo de residuos y plásticos difieren considerablemente entre los países del mundo. A su vez, estos factores influyen en la cantidad de plástico que entra en los sistemas fluviales [3].

Ahora bien, toda esta problemática hasta el momento se ha abordado desde el punto de vista de la superficie del tema, sin embargo, hay un entorno que se ve muy afectado y es esencial para nuestra vida y la vida marina: los arrecifes de coral. Los arrecifes de coral se ven amenazados por un aumento de las tensiones derivadas de las actividades humanas, como el desarrollo costero, la contaminación, la sobrepesca, las prácticas pesqueras destructivas y la actividad turística [4]. Toda esta problemática afecta en gran manera estos ecosistemas, y es que, la importancia de estos radica principalmente en su singularidad, la resiliencia de sobrevivir en condiciones inadecuadas (como las que actualmente enfrenta) y su capacidad de aflorar grandes alojamientos de agua fría, lo que mantiene un balance en la temperatura de los mares.

Por esto, se acude a un gran campo que se encuentra (a pesar de estar en etapas tempranas) en constante desarrollo, innovación e implementación para contribuir a la solución de problemáticas de este tipo: La robótica. El desarrollo del campo de la tecnología de robots móviles ha ampliado con éxito áreas que no se habían explorado antes, porque el avance de los últimos prototipos de robots en este campo ha mejorado sus funciones, lo cual es muy obvio en sistemas terrestres, acuáticos y aéreos.

Hoy en día, muchos de los prototipos de robots que se utilizan en entornos marinos comprometedores para el ser humano tratan de emular, principalmente, la morfología de animales acuáticos. La forma de los animales acuáticos es fundamental, pues hay que considerar su capacidad para moverse en el medio submarino, lo que supone un desafío para reproducir su dinámica. Sin embargo, el entorno marino tiene una diversidad de factores a considerar que dificultan la tarea de realizar con facilidad las mismas, y muchos sensores operan con dificultad debido a que las ondas no atraviesan con facilidad estas profundidades de aguas. El entorno submarino se encuentra bajo una condición limitada en la que la onda de radio no pasa. Uno de los sensores que proporcionan información útil al robot es un sistema de cámaras ópticas, pero su uso es limitado debido a la baja visibilidad y a las condiciones de poca luz del medio subacuático [5].

Para realizar con éxito el siguiente trabajo, se toman elementos clave de tres clases de robots: terrestres, aéreos y submarinos. De los terrestres se toman en consideración los rápidamente incrementa. Es por eso que la regulación del uso de plásticos y demás materiales invasivos al medioambiente factores que asegurarán un éxito tanto comercial como funcional: Para que los robots terrestres tengan éxito comercial, son atributos esenciales un alto grado de autonomía, un bajo coste de fabricación y mantenimiento, así como la minimización de las limitaciones de despliegue en diferentes entornos [6].

En cuanto a los robots aéreos, el enfoque se realiza sobre un robot previamente diseñado con la capacidad de detectar y seguir un objetivo mediante una cámara. Uno de los casos más desafiantes en el SAR utilizando cuadricópteros de vehículos aéreos no tripulados es el seguimiento de un objeto que acaba de ser reconocido... Una vez reconocido el objeto, el cuadricóptero puede rastrear y seguir el objeto con un alto grado de precisión [7].

Y, a la hora de analizar los robots submarinos operados remotamente, es esencial contar con herramientas que faciliten el estudio de los mismos, principalmente análisis que faciliten estudiar y mejorar los diseños estructurales de los robots. Con el desarrollo de la tecnología de simulación por ordenador, la dinámica de fluidos computacional (CFD) se ha utilizado ampliamente en el cálculo de la simulación hidrodinámica [8].

\section{MARCO TEÓRICO}

Para el desarrollo de sistemas mecatrónicos, existe un modelo más flexible que se puede seguir. Se basa en tres elementos: el modelo en forma de $\mathrm{V}$ a nivel macro, el ciclo general de resolución de problemas a nivel micro, y finalmente el módulo que repite los pasos de operación en el diseño del sistema mecatrónico.

\section{A. Modelo en $V$}

$\mathrm{El}$ modelo en forma de $\mathrm{V}$ se ha establecido firmemente en el campo de la ingeniería de software y desde entonces se ha adaptado a las necesidades de la mecatrónica. El método $\mathrm{V}$ es una representación gráfica del ciclo de vida de desarrollo del sistema. Resume los principales pasos a seguir para verificar los entregables correspondientes del sistema. Para el siguiente proyecto, se tomó esta metodología como base para realizar cada uno de los sistemas con lo conforman.

\section{B. Software}

Independientemente del aspecto de la implementación del robot, la programación de sus funciones es fundamental para la correcta realización de sus tareas. La correcta integración de las órdenes programadas en el procesador y la comunicación con el mundo exterior a través de sensores o señales adquiridas son la base para el correcto funcionamiento de cualquier sistema "inteligente". Para la investigación actual para la programación, diseño estructural y diseño electrónico de un robot acuático, se implementaron diferentes softwares como el entorno de desarrollo integrado de Arduino, Microsoft Visual Studio, SolidWorks y Proteus Design Suite para el diseño y prototipaje correcto de los componentes a utilizar. 


\section{Microprocesador}

Los microprocesadores poseen procesadores, memoria y diferentes puertos para entradas y salidas $(\mathrm{E} / \mathrm{S})$ todo integrado en sí mismo. Para el siguiente proyecto, se basó la programación en el microcontrolador ATmega328P, por medio del cual se cumplen dos funciones principales: La primera es controlar las turbinas que darán dirección al robot y segundo, la conexión de la cámara con inteligencia artificial programada para la detección de objetos sobre la superficie del agua y simplificar el manejo del navío a distancias mayores.

\section{Inteligencia Artificial}

La inteligencia artificial (IA) se implementa en el proyecto a realizar por medio de redes neuronales, es una disciplina científica relacionada con los programas informáticos, que tiene como objetivo emular sus facultades intelectuales propias y humanas en sistemas artificiales. Las redes neuronales en las últimas décadas han transformado el método de procesamiento de la información en el que, a través de instrucciones específicas almacenadas en la memoria, pueden tomar decisiones basadas en ellas. Pueden implementar técnicas de aprendizaje y organización para detectar patrones, márgenes específicos e incluso adaptarse a los cambios generados en el sistema. La visión artificial como parte del campo de la IA se utiliza para diferentes propósitos: segmentación de imágenes, detección de objetos, reconocimiento facial, detección de patrones, clasificación de imágenes y muchas otras técnicas más avanzadas como las que se utilizan hoy en día para los coches autónomos. Es por ello que es una parte fundamental para la fabricación del prototipo.[9]

\section{METODOLOGÍA}

Para este proyecto se identificó que la metodología más adecuada seria la metodología en $\mathrm{V}$, debido a que posee una amplia variedad de sistemas que se pueden dividir en diferentes subsistemas. Este tipo de metodología es ideal para el diseño y la realización de proyectos del área de la robótica y es la metodología correcta para productos mecatrónicos. Para este proyecto seguiremos los pasos mostrados en la figura 1 la cual nos muestra un camino de cómo llevar un orden desde el diseño con sus requerimientos iniciales y luego realizando pruebas en cada sistema por separado se logra llegar a realizar una integración final para poder juntar todos los sistemas y realizar un producto completo garantizando su funcionamiento con todas las especificaciones requeridas.

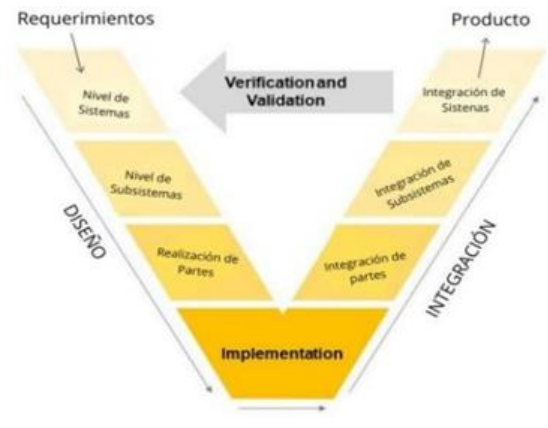

Figura 1. Metodología "V"

Los resultados a evaluar en este proyecto son cuantitativos ya que con estos se pueden evaluar las características del robot. Durante la realización de este proyecto las variables a considerar y evaluar son: Eficiencia energética como variable independiente y las variables dependientes que son, precisión de detección, velocidad de motores, longitud de respuesta.

\section{DESARROLLO}

Principalmente antes de empezar a desarrollar la metodología se necesita definir cuáles serán los requerimientos del proyecto, mismos que ayudaran a definir los sistemas principales y se podrá planear cómo será la forma más factible para desarrollar dicho producto, en el caso de este proyecto se identificaron una serie de requerimientos enfocados al correcto funcionamiento del mismo, como ser una estructura capaz de resistir la corrosión y que fuese $100 \%$ impermeable para poder almacenar todos los subsistemas dentro de ella de forma segura.

\section{A. Etapa 1 - Nivel de los sistemas - Ciclo:}

Siguiendo los pasos de la metodología el primer paso a seguir para desarrollar un producto mecatrónico es definir los sistemas del proyecto, se definieron 3 sistemas principales:

- Sistema mecánico, en él se observa la parte de diseño de estructura, análisis de flujo del agua y la capacidad de resistir la corrosión que causa el agua salada en la mayoría de los materiales.

- Sistema electrónico, el cual se compone de nuestro microcontrolador ATmega328P, controladores de velocidad para motores sin escobillas, componentes de comunicación por radiofrecuencia y baterías recargables para alimentar el sistema electrónico.

- Sistema de visión, en este se encuentra el uso de una cámara FPV el cual por medio de una red neuronal es capaz de detectar objetos en tiempo real.

\section{B. Etapa II: Nivel de los subsistemas}

Después de definir cada sistema se continua a determinar los subsistemas

Sistema Mecánico:

1. Subsistema Mecánico, Se realizaron diseños CAD y pruebas de la estructura que pudiera cumplir con la función, se decidió utilizar como base el diseño de un

$19^{\text {th }}$ LACCEI International Multi-Conference for Engineering, Education, and Technology: "Prospective and trends in technology and skills for 
navío catamarán que consta de 2 cascos, permitiéndonos utilizar el orificio que se genera entre ambos cascos como compartimiento para los desechos marinos. Se implemento un diseño que nos permitirá mantener la electrónica sobre la superficie del agua dejándonos una posición segura por cualquier fuga de agua que pueda quedar al momento de fabricar la estructura.

2. Subsistema de Materiales, se decidió que el material más adecuado para este proyecto sería láminas de PVC, al ser un material resistente al agua y siendo un termoplástico es posible moldearlo de la manera en que necesitamos económicamente. El PVC es mucho más liviano que otros materiales metálicos y el precio es más bajo es por ello que se decidió utilizar láminas de PVC de 4 x 8 pies y un grosor de $13 \mathrm{~mm}$ y de $6 \mathrm{~mm}$.

\section{Subsistema Electrónico:}

1. Subsistema de comunicación, para poder utilizar todo a distancia fue necesario utilizar una comunicación por medio de la radio frecuencia, se utilizará recibidores de señal de $2.4 \mathrm{GHz}$ y de $5.8 \mathrm{GHz}$ para poder recibir señal del control remoto y de la cámara. Se utilizaron 2 canales diferentes para no tener choques de señal. Una placa electrónica donde se montará el microcontrolador.

2. Subsistema electrónico, se diseñó una placa electrónica que alberga el microcontrolador ATmega328P y le permite comunicarse con los demás componentes, con todas sus salidas y entradas de señal.

3. Subsistema de alimentación, se utilizarán baterías de polímero de litio recargables para poder alimentar todo el circuito de fuerza. El voltaje de las baterías puede variar dependiendo de los motores que se utilicen y la potencia máxima que se desea alcanzar. También se utilizará una batería recargable que genere $5 \mathrm{v}$ para poder alimentar el circuito de control.

4. Subsistema de propulsión, se utilizarán 2 propulsores a prueba de agua, los cuales por medio de señales PWM enviadas desde el control remoto hasta el microcontrolador por medio de radiofrecuencia y se procede a enviar a sus controladores de velocidad. Las turbinas utilizadas tienen un rango desde los $12 \mathrm{v}$ hasta los $22 \mathrm{v}$ y se decidió alimentarlas con $14.8 \mathrm{v}$. Los autores hondureños de "Design of an Underwater Robot for Coral Reef Monitoring in Honduras" utilizaron este mismo tipo de turbinas para su robot submarino el cual daba la fuerza necesaria para poder movilizarse bajo el agua, se menciona como al integrar 4 turbinas lograron obtener 3 grados de libertad para maniobrar de excelente manera el robot submarino.[10]

\section{Subsistema de Visión}

1. Subsistema de Detección, se integrará una cámara que envía imagen de video por medio de radiofrecuencia en tiempo real hacia un recibidor que se conecta a un computador para poder ser enlazado a distancia sin utilizar cableado. El propósito principal será detectar objetos como botellas de plástico para apoyar la visión del operador a larga distancia y acertar al momento de recolectar los desechos.

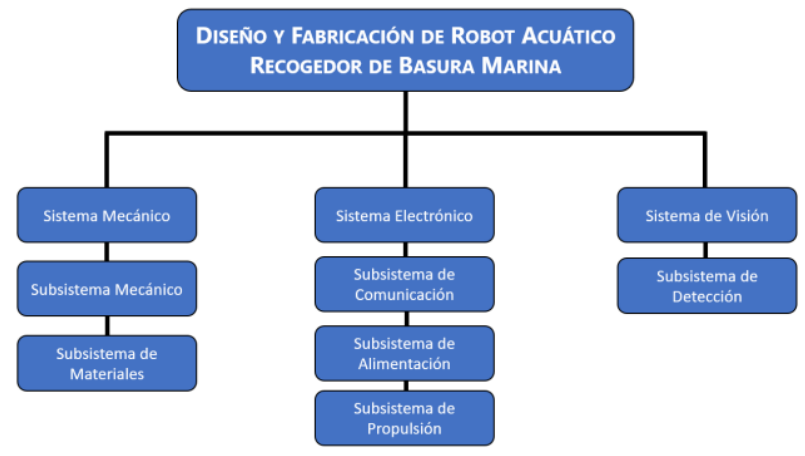

Figura 2. Sistemas y Subsistemas del robot acuático

\section{Etapa III: Realización de Partes}

Una vez tomado en cuenta cada subsistema se comenzó con la elaboración de las partes de cada subsistema comenzando con la parte mecánica.

Partes Mecánicas:

- Se mecanizaron 2 láminas de PVC de 4x8 pies de $13 \mathrm{~mm}$ de grosor, se realizaron los cortes del diseño propuesto y se utilizaron técnicas de termoformado para poder moldear el material de una manera adecuada para poder realizar el diseño de la figura 3 .

- Se fabrico una caja de PVC de 10x10x5 pulgadas para almacenar el panel de control con la electrónica del vehículo.

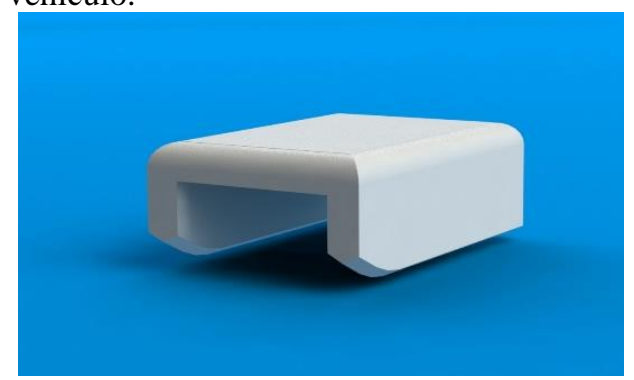

Figura 3. Estructura renderizada del prototipo

- Se fabrico un housing por medio de impresión 3D para poder acoplar la cámara de video en la parte superior del prototipo que cuenta con una tapadera que se acopla a presión.

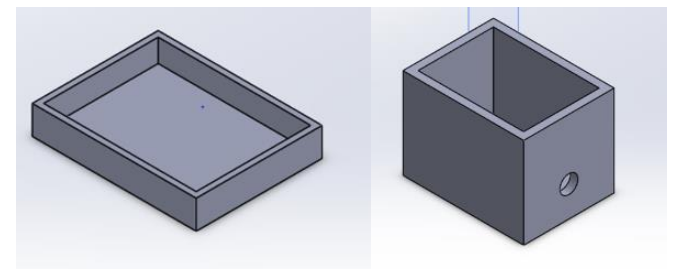

$19^{\text {th }}$ LACCEI International Multi-Conference for Engineering, Education, and Technology: "Prospective and trends in technology and skills for sustainable social development" "Leveraging emerging technologies to construct the future", Buenos Aires -Argentina, July 21-23, 2021. 


\section{Figura 4. Diseño en SolidWorks de housing para cámara}

Subsistema electrónico:

- Se realizaron pruebas físicas de los componentes en una placa de prototipaje para evaluar el funcionamiento adecuado. Probando cada canal con sus respectivas señales.

- Se determino que las baterías a utilizar serian de $14.8 \mathrm{~V}$ $9000 \mathrm{mAh}$ para poder utilizar nuestro sistema de propulsión ya que los motores son de ese voltaje

- Se desarrollo una programación en el software de Arduino capaz de conectar los 3 sistemas utilizando el control remoto del robot con sus diferentes canales con un funcionamiento como en la figura 5.

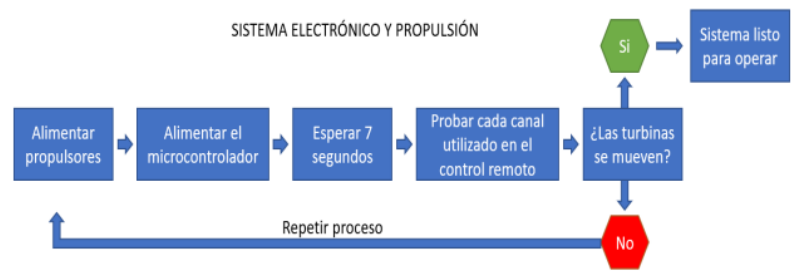

Figura 5. proceso para verificar el funcionamiento del sistema de propulsión y electrónico

- Se realizo una placa en el software Proteus la cual servirá como adaptador para nuestro microcontrolador. Consta de 30 pines hembra que serán conectados a los 30 pines macho que tiene el microcontrolador, cada pin tiene su pista que estará conectado a una bornera para poder recibir y enviar señal por medio de ellas. Se realizo de esta manera para poder tener todos los pines listos para poder ser utilizados en el futuro para poder instalarle mejoras al primer prototipo.
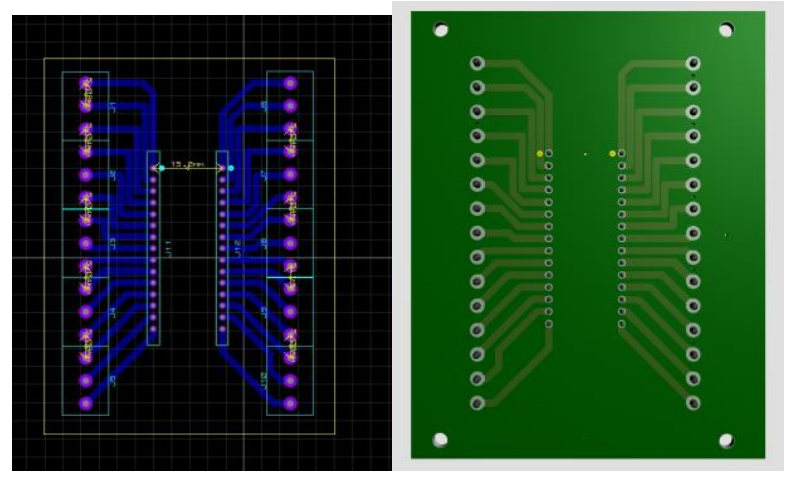

Figura 6. Diseño de placa en Proteus

- Se decidió utilizar cableado calibre 12 ya que el consumo máximo de corriente será de $22 \mathrm{~A}$ el cual es un amperaje adecuado para este calibre de cable.

Subsistema de Detección
- Se realizo una comunicación eficiente entre la cámara FPV y la computadora por medio de un receptor de radiofrecuencia $5.8 \mathrm{GHz}$ el cual capta la señal como una cámara web.

- Se realizo una programación capaz de transmitir la señal en tiempo real y con la capacidad de tomar captura de imágenes y redimensionarlas para poder enviarlas ya sea a sus carpetas positivas y negativas.

- Se tomaron 850 fotos negativas de fondos en los cuales podría trabajar el prototipo como ambientes acuáticos con colores parecidos al agua que se encuentra en mares, ríos y lagos.

- Se tomaron 100 fotos positivas como muestra la figura 7 con varios lados de la botella y con objetos parecidos para detectar otros objetos que se parezcan dentro del agua.

- El sistema funciona de la siguiente manera: primero se debe realizar la programación en Visual Studio para desarrollar la aplicación de abrir la imagen de video y poder capturar imágenes por medio de la librería Open CV en el software de Visual Studio Code como la utilizan en la investigación de autores hondureños "Study Case: Teleoperated Voice Picking Robots prototype as a logistic solution in Honduras" que al utilizar open CV logran implementarle visión artificial al prototipo que crearon de igual forma como se utiliza en nuestro ROV recogedor de basura marina con la diferencia que no se utiliza opciones de voz [11]. En el caso de este proyecto se realizó programación en lenguaje Python por su buen desempeño para implementar sistemas de visión artificial. Previamente se debió instalar las librerías necesarias como lo es la librería imutils que funciona al momento de redimensionar las imágenes, esta función se implementa para que el tamaño de las imágenes no sea tan pesado y que la aplicación de entrenamiento de red neuronal pueda desarrollar su trabajo de forma eficiente en un periodo de tiempo corto.

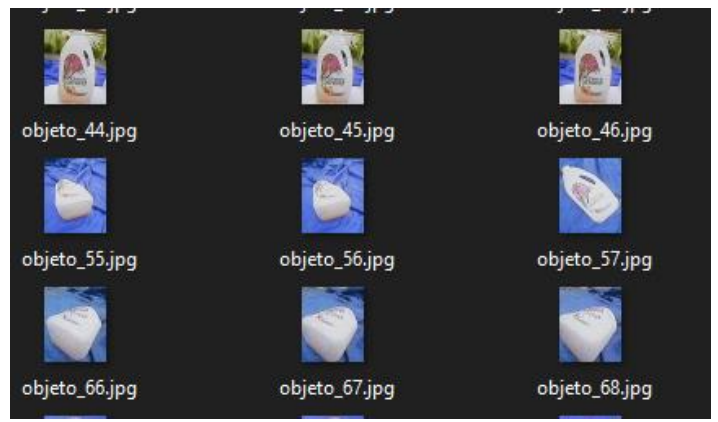

Figura 7. Imágenes variadas del objeto a detectar

- Una vez teniendo las imágenes se pudo utilizar la aplicación de cascade trainer que nos realiza la función de entrenamiento clasificando imágenes positivas y negativas, una vez entrenada la red nos dará un archivo .XML que nos permitirá mandarlo a llamar cuando 
realizamos la segunda programación que esta es la que vera el operador al momento de utilizar el sistema de visión. Esta segunda programación nos manda a llamar la imagen de video en tiempo real y se programa un recuadro que encerrara el objeto deseado, también se le programa un título al rectángulo para poder nombrar lo que se esta detectando. En el caso de este proyecto se decidió titular el rectángulo como objeto ya que, se está detectando múltiples tipos de botellas plásticas que puedan estar en el agua. Para detectar el objeto la programación convierte la imagen en escala de grises e intenta encontrar patrones relacionado a los patrones de las imágenes positivas con la cual se entrenó.

\section{Etapa IV: Integración de Partes}

Siguiendo la metodología de estudio se procedió a realizar pruebas a los componentes que se utilizan en cada subsistema

\section{Integración de partes mecánicas}

- Cada lamina del robot fue ensamblada con tornillos y entre cada lamina iba una capa de pegamento para PVC el cual nos sirvió como sellador entre las grietas que se formaban. Se utilizo el termoformado para darle las curvaturas deseadas a las láminas de abajo y de arriba en los costados el cual nos permite darle ese diseño al vehículo para una mejor fluidez dentro del agua y una mejor aerodinámica. Para terminar de sellar las piezas se decidió utilizar masilla para vehículos la cual se le implemento una capa protectora en cada grieta para un mejor sellado. En la figura 8, se muestra como la estructura esta casi lista con los detalles azulados que son los puntos donde fue aplicada la masilla.

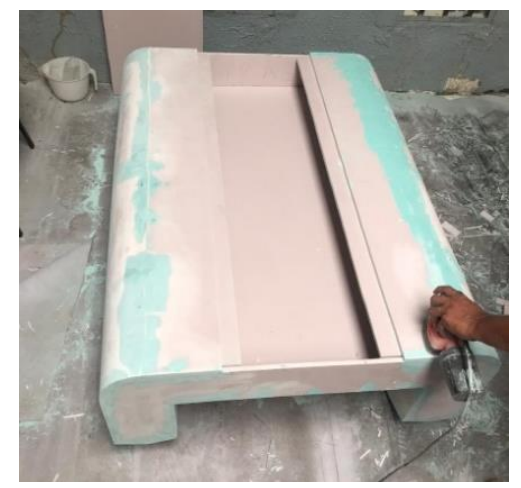

Figura 8. Estructura final sin la tapadera superior

- Se utilizo una malla metálica de aluminio galvanizado para crear el compartimiento de la basura, se instaló por medio de tornillos de acero inoxidable forrados con teflón y sellados con pegamento para PVC para evitar cualquier filtración.

- Se imprimió el housing de la cámara de video y se incorporó en la estructura como se muestra en la figura 8.

- La estructura se introdujo al agua para poder ver si tenía fugas y así poder ser selladas, pero con las defensas implementadas se logró obtener una estructura completamente impermeable.

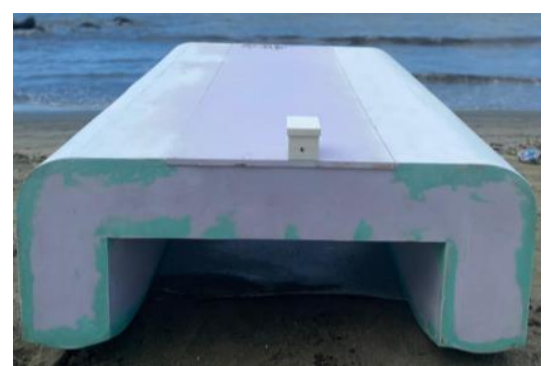

Figura 8. Estructura con housing y tela metálica incorporadas

2. Integración de partes electrónicas

- Se realizó un panel de control como lo muestra la figura 9. con medidas de $10 \times 10 \times 5$ pulgadas que alberga el microcontrolador ATmega328P con su respectivo adaptador, 1 receptor de señal de radiofrecuencia $2.4 \mathrm{GHz}, 2$ controladores de velocidad de motores sin escobillas, 1 relé que controla el encendido y apagado de la cámara, 2 baterías de litio utilizadas para alimentar el circuito de fuerza y una batería recargable con salida de $5 \mathrm{v}$ para alimentar el circuito de mando.

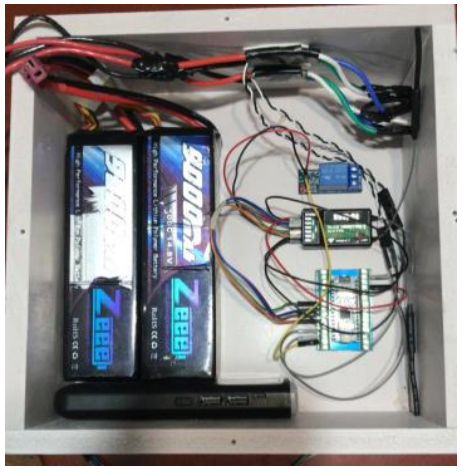

Figura 9. Panel de control con sus componentes integrados.

- Se realizaron pruebas de control a distancia y se llegó a la conclusión que a 700 metros la señal se debilita y empieza a tener pequeñas fallas para poder maniobrar bien el robot dentro del agua.

3. Integración de partes de Visión

- Se realizaron pruebas de detección con la red neuronal que se entrenó, dando buenos resultados a la hora de detectar botellas plásticas de color blanco en condiciones de mucha y de poca iluminación. Como muestra la figura 10, se observa como el sistema puede detectar varios objetos en tiempo real en una misma imagen. 


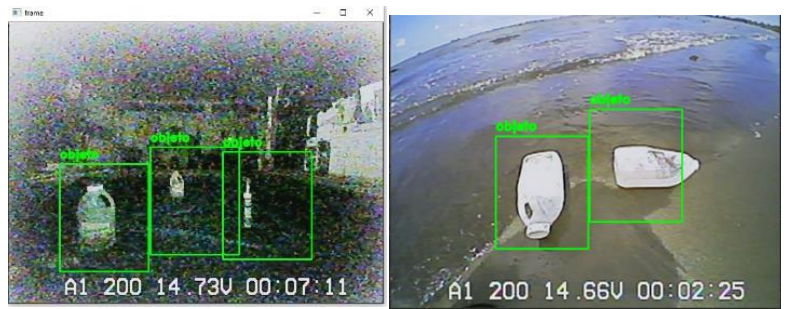

Figura 10. Pruebas de detección en ambientes de poca y mucha iluminación

\section{E. Etapa V: Integración de Subsistemas}

En esta etapa cada subsistema fue integrado en su sistema principal para poder ser posteriormente integrado en el producto final al juntar cada sistema.

\section{Integración de subsistemas mecánicos}

- La estructura se introdujo al agua para poder ver si tenía fugas y así poder ser selladas, pero con las defensas implementadas se logró obtener una estructura completamente impermeable y capaz de flotar y mantenerse estable con cualquier movimiento que realice el agua de forma natural.

Integración de subsistema electrónico

- $\quad$ se observó un funcionamiento adecuado de la respuesta del control remoto tanto en los motores como en el encendido y apagado de la cámara de video.

Integración de subsistema de visión

- Se observo una buena detección en el sistema y se definió que estaba listo para ser implementado en el producto final.

\section{F. Etapa VI: Integración de Sistemas}

En esta etapa del proyecto ya se tienen los 3 sistemas principales funcionando por separado, una vez teniéndolos listos se puede comenzar con la parte de integrar los sistemas en un mismo producto para llegar a nuestro objetivo.

\section{Integración de Sistema Electrónico Con Sistema Mecánico}

1. una vez teniendo lista la estructura y el sistema mecánico se logró acoplar los motores, los cuales fueron instalados en la parte trasera en sus costados exteriores para poder girar el vehículo dependiendo del lado en el que se active el motor.

2. Una vez instalados los motores se instaló el panel de control en la parte interna del vehículo y se cablearon los motores con los controladores de velocidad que van conectados directamente a las baterías de litio y que son controlados por el microcontrolador.

3. Se probo que cada motor girara de manera correcta con la tapadera cerrada para comprobar que la señal no se perdiera $y$ se mantuvo estable y con una buena manejabilidad.

Integración de Sistema de Visión Con Los Demás Sistemas

1. Se incorporo la cámara de video en el housing correspondiente

2. Se cableo de manera correcta la cámara al relé por dentro de la estructura.

3. Se probo la respuesta del control para el encendido y apagado de la cámara de video.

\section{RESULTADOS FINALES}

Siguiendo los pasos realizados en la metodología se logró diseñar y fabricar un prototipo final capaz de recolectar basura marina y detectar objetos por medio de un sistema de visión artificial. En la figura 11, se puede apreciar el prototipo navegando en el mar de Omoa, Cortes, en un puerto donde descansan navíos. Se realizaron pruebas de manejo, de recolección y de visión, cabe mencionar que todas las pruebas fueron pasadas con éxito dejándonos un prototipo capaz de realizar las tareas para las cuales fue creado. Se hizo un presupuesto final del gasto total del prototipo y el precio de construcción y componentes ronda por los 26,000 lempiras el cual es equivalente a 1,075 \$ USD.

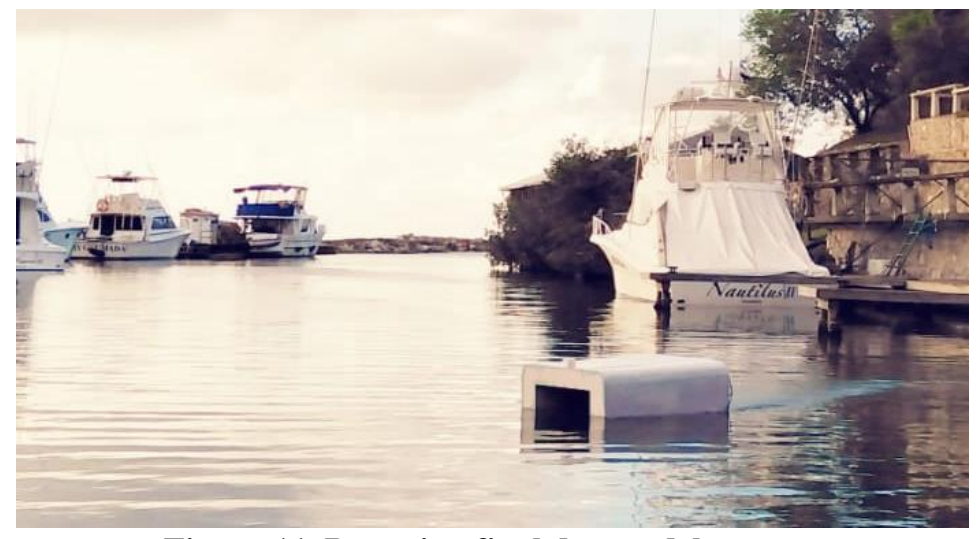

Figura 11. Prototipo final dentro del agua

\section{CONCLUSIONES}

El prototipo final con la integración de sus 3 sistemas principales cumple con todos los requerimientos que se plantearon antes de realizar el proyecto, cumple con su buen control dentro del agua. Utilizando el diseño de los navíos catamarán se logra tener una estructura capaz de recolectar basura que flota en las superficies, dando una capacidad volumétrica de recolección de 0.25 metros cúbicos de basura marina. Como se muestra en la figura 11, se puede observar el vehículo navegando en un puerto donde se realizaron pruebas de recolección de desechos y fueron un éxito. No solamente es capaz de recolectar basura, así mismo, posee una manejabilidad espectacular, toma unos pocos minutos de uso para que una persona corriente logre acostumbrarse al control del vehículo dentro del agua. El sistema de visión artificial con detección de objetos permite a los operadores poder visualizar desde lejos lo que el robot mira en primera persona y como ayuda extra, la red neuronal se encarga de detectar objetos para que el operador pueda observarlos con mayor facilidad. 


\section{REFERENCIAS}

[1] U. N. E. Programme, «Addressing Marine Plastics: A Systemic Approach - Recommendations for Action», 2019, Accedido: ene. 23, 2021. [En línea]. Disponible en:

https://wedocs.unep.org/xmlui/handle/20.500.11822/31642

[2] «RIOS DE GUATEMALA».

http://www.insivumeh.gob.gt/hidrologia/rios\%20de\%20guate.htm\#PRINCIPAL ES\%20RIOS\%20DE\%20GUATEMALA (accedido ene. 23, 2021).

[3] C. J. van Calcar y T. H. M. van Emmerik, «Abundance of plastic debris across European and Asian rivers», Environ. Res. Lett., vol. 14, n. ${ }^{\circ} 12$, p. 124051, dic. 2019, doi: 10.1088/1748-9326/ab5468.

[4] C. K. Tito y E. E. Ampou, «Coral reefs ecosystem degradation at Nusa Penida, Bali», IOP Conf. Ser. Earth Environ. Sci., vol. 429, p. 012053, ene. 2020, doi: 10.1088/1755-1315/429/1/012053.

[5] B. Kim, J. Kim, M. Lee, M. Sung, y S. Yu, «Active Planning of AUVs for 3D Reconstruction of Underwater Object using Imaging Sonar», en 2018 IEEE/OES Autonomous Underwater Vehicle Workshop (AUV), nov. 2018, pp. 1-6. doi: 10.1109/AUV.2018.8729706.

[6] Y. Chen, M. Zhang, D. Hong, C. Deng, y M. Li, «Perception System Design for Low-Cost Commercial Ground Robots: Sensor Configurations, Calibration, Localization and Mapping», en 2019 IEEE/RSJ International Conference on Intelligent Robots and Systems (IROS), nov. 2019, pp. $6663-$ 6670. doi: 10.1109/IROS40897.2019.8968078.

[7] A. Haris Indra Fadhillah, A. Taqwim Safrudin, y S. Darma, «Unmanned aerial vehicle object tracking and following», J. Phys. Conf. Ser., vol. 1528, p. 012018, abr. 2020, doi: 10.1088/1742-6596/1528/1/012018.

[8] Z. Wang, Y. Chen, T. Wang, y B. Zhang, «Design and hydrodynamic simulation analysis on debris cleaning robot underwater», J. Phys. Conf. Ser., vol. 1550, p. 022004, may 2020, doi: 10.1088/1742-6596/1550/2/022004.

[9] A. Sorto, T. Marquez, A. Carrasco, y J. Ordoñez, «Face Recognition and Temperature Data Acquisition for COVID-19 Patients in Honduras», $J$. Phys. Conf. Ser., vol. 1710, p. 012009, nov. 2020, doi: 10.1088/17426596/1710/1/012009.

[10] J. L. O. Avila, M. G. O. Avila, y M. E. Perdomo, «Design of an Underwater Robot for Coral Reef Monitoring in Honduras», en 2021 6th International Conference on Control and Robotics Engineering (ICCRE), abr. 2021, pp. 86-90. doi: 10.1109/ICCRE51898.2021.9435710.

[11] J. L. O. Avila et al., «Study Case: Teleoperated Voice Picking Robots prototype as a logistic solution in Honduras», en 2020 5th International Conference on Control and Robotics Engineering (ICCRE), abr. 2020, pp. 19-

24. doi: 10.1109/ICCRE49379.2020.9096483.

$1^{\text {th }}$ LACCEI International Multi-Conference for Engineering, Education, and Technology: "Prospective and trends in technology and skills for sustainable social development" "Leveraging emerging technologies to construct the future", Buenos Aires -Argentina, July 21-23, 2021. 